\title{
Análise química e microbiológica da água em pontos de coleta distintos
}

Carla Gabriela Azevedo Misael* (Graduanda em Engenharia Química na Universidade Federal de Campina Grande - UFCG);

Caroline Vasconcelos Fernandes (Graduanda em Engenharia Química na Universidade Federal de Campina Grande - UFCG);

Filipe José Ferreira Chaves (Graduando em Engenharia Química na Universidade Federal de Campina Grande UFCG);

Josiele Souza Batista Santos (Graduanda em Engenharia Química na Universidade Federal de Campina Grande UFCG);

Juan Nicolas Andrade Cavalcante (Graduando em Engenharia Química na Universidade Federal de Campina Grande - UFCG);

Suênia Fernandes de Vasconcelos (Graduanda em Engenharia Química na Universidade Federal de Campina Grande - UFCG).

*Email:carla.misael94@gmail.com

Resumo:

A água constitui um dos principais requisitos para existência e manutenção da vida no planeta. Apesar de sua abundância, a maior parte da água disponível encontra-se imprópria para consumo, sendo por motivos naturais como o excesso de sal, ou por motivos ocasionados pelo ser humano como a poluição. Esta poluição por vezes é perceptível, contudo em grande parte só é descoberta por meio de análises específicas e precisas. Diversos órgãos públicos visam controlar a potabilidade da água fornecida em redes domésticas, estabelecendo para isso parâmetros a serem cumpridos. Logo, o presente trabalho buscou verificar se a água fornecida nos municípios de Campina Grande, Cuité e Picuí, localizados no Estado da Paraíba, correspondia qualitativamente ao esperado. Para tanto, analisou-se fatores físicoquímicos como $\mathrm{pH}$, alcalinidade e dureza da água, através de aparelhos específicos e técnicas de titulometria. Fatores microbiológicos também foram analisados a fim de verificar a presença de coliformes totais e coliformes fecais. Todas as amostras seguiram por um processo de coleta estéril, e apresentaram níveis toleráveis para os fatores pontuados, sendo então próprias para o uso doméstico.

\section{Palavras-chave:}

Água; potabilidade; alcalinidade; análise microbiológica 


\section{I ntrodução}

É de conhecimento comum a importância da água para os processos vitais e para a saúde humana. A água é essencial para o funcionamento biológico em todos os níveis, desde o metabolismo dos organismos vivos até o equilíbrio dos ecossistemas. Contudo, estima-se que cerca de 1,5 bilhões de pessoas não tenham, em todo o mundo, acesso a água de boa qualidade, e $10 \%$ da carga global de doenças seja devida à má qualidade da água e a deficiências na disposição de excretas e na higiene (Prüss-Ustin et al., 2008; Confalonieri et al., 2010).

A potabilidade da água é composta por aspectos físicos, químicos e biológicos, destacando-se dentre os parâmetros microbiológicos, os coliformes. Por definição, coliformes são bacilos aeróbicos e/ou anaeróbios facultativos, Gram-negativos, não esporulados, capazes de fermentar a lactose com produção de ácido e gás (Novak et al., 2001). Pertencem a este grupo predominantemente, bactérias dos gêneros Escherichia, Citrobacter, Klebsiella e Enterobacter. Dessas, apenas a Escherichia coli tem como habitat primário o trato intestinal do ser humano e animais. Os demais, além de serem encontrados nas fezes, também estão presentes em outros ambientes como vegetais e solo. Consequentemente, a presença de coliformes totais não indica, necessariamente, contaminação fecal recente ou ocorrência de enteropatógenos (Dias, 2008).

Em seu percurso natural entre o solo e rochas, a agua dissolve inúmeros sais. Porém, por vezes ela adquire uma quantidade elevada de íons $\mathrm{Ca}^{+2} \mathrm{e} \mathrm{Mg}^{+2}$, caracterizando o que se define por 'água dura'. Esses íons reagem com o sabão criando sais insolúveis em água, consequentemente o sabão perde o poder de limpeza e os sais insolúveis aderem a superfícies, causando problemas em escala industrial. Além disso, quando o cálcio e o magnésio são ingeridos em doses elevadas, podem acarretar doenças ao ser humano, como a osteoporose, pedras nos rins, hipertensão, dentre outros. A dureza divide-se em dureza temporária e dureza permanente. A primeira é gerada pela presença de carbonatos e bicarbonatos e pode ser eliminada por meio de fervura da água. A segunda é devida a cloretos, nitratos e sulfatos, os quais não são suscetíveis à fervura. À soma da dureza temporária e permanente dá-se o nome de "dureza total” da água (Lee, 1999).

A dureza pode ser expressa em $\mathrm{mg} / \mathrm{L}$ de carbonato de cálcio $\left(\mathrm{CaCO}_{3}\right)$, onde valores acima de $180 \mathrm{mg} / \mathrm{L}$ de $\mathrm{CaCO}_{3}$ podem induzir à formação de incrustações nas canalizações e valores inferiores a $60 \mathrm{mg} / \mathrm{L}$ podem sugerir fenômenos corrosivos nos órgãos do sistema de abastecimento de água. O decreto-lei ${ }^{\circ}$ 306/2007, de 27 de agosto, que estabelece o regime da qualidade da água destinada ao consumo humano, tendo por objetivo proteger a saúde humana dos efeitos nocivos resultantes da eventual contaminação dessa água, recomenda na nota 4 parte III, que a dureza total em carbonato de cálcio esteja compreendida entre $150 \mathrm{mg} / \mathrm{L}$ e $500 \mathrm{mg} / \mathrm{L}$ de $\mathrm{CaCO}_{3}$ (Comissão Especializada da Qualidade da Água, 2012).

Entre as impurezas encontradas na água, existem aquelas que são capazes de reagir com ácidos, podendo neutralizar certa quantidade desses reagentes. Essas impurezas conferem às águas a característica de alcalinidade. A alcalinidade é devida principalmente à presença de bicarbonatos, carbonatos e hidróxidos, sendo mais comuns os seguintes compostos: hidróxidos de cálcio ou de magnésio, carbonatos de cálcio ou de magnésio, bicarbonatos de cálcio ou de magnésio, bicarbonatos de sódio ou de potássio. Valores muito elevados de alcalinidade podem ser indesejáveis em uma água a ser utilizada para fins industriais, uma vez que podem ocasionar problemas de formação de depósitos e corrosão, de acordo com a utilização desta água (Oliveira, 2007).

Tendo em vista todo o contexto abordado, este trabalho objetiva analisar amostras de águas colhidas de três diferentes cidades da Paraíba acerca de sua alcalinidade, dureza e composição microbiológica

\section{Metodologia}

Para a realização das análises, recolheu-se amostras das cidades de Campina Grande (A1), Cuité (A2) e Picuí (A3), que tem como fontes de abastecimento o Açude do Boqueirão, poços particulares e o Açude de Várzea Grande, respectivamente. A assepsia foi realizada nos pontos de coleta com etanol 70\%, 
deixando a água correr por cerca de dois minutos antes da coleta. Todos os frascos utilizados para captação da água encontravam-se estéreis.

Na análise microbiológica utilizou-se $100 \mathrm{~mL}$ de cada amostra, adicionando-se o Colitag ${ }^{\circledR}$ nos frascos, que permaneceram em estufa à $35^{\circ} \mathrm{C}$ por um período de 24h. Posteriormente, os frascos foram inspecionados visualmente à luz ambiente e com lâmpada UV.

Afim de determinar a alcalinidade, separou-se $25 \mathrm{~mL}$ de água e adicionou-se 2 gotas de fenolftaleína, observando-se a coloração. Caso a solução tivesse a coloração alterada, titulava-se a amostra com ácido sulfúrico $\left(\mathrm{H}_{2} \mathrm{SO}_{4}\right)$ 0,02N, definindo o volume de ácido gasto como P; do contrário, $\mathrm{P}$ era definido como zero. Logo após, adicionou-se 2 gotas de metilorange $0,1 \%$, e iniciou-se a titulação com uma solução de ácido sulfúrico $\left(\mathrm{H}_{2} \mathrm{SO}_{4}\right)$ 0,02N, até a completa mudança de coloração. Todas as amostras foram analisadas em triplicatas.

A dureza da água foi determinada através de outro método analítico, onde $3 \mathrm{~mL}$ de soluçãotampão e 2 gotas de negro de eriocromo foram respectivamente adicionados à $25 \mathrm{~mL}$ de água. Em seguida, a amostra foi titulada com uma solução de EDTA-Na2 $0,025 \mathrm{~N}$ até a mudança de coloração. A análise foi realizada em triplicata. Verificou-se o pH de todas as alíquotas de água.

\section{Resultados e discussão}

$\mathrm{O}$ pH das amostras foi verificado por 8 vezes, afim de se obter uma média com segurança. Os resultados estão expostos na Tabela 1.

Tabela 1 - Valores de $\mathrm{pH}$ nas diferentes amostras.

\begin{tabular}{cccccccccc}
\hline Amostras/pH & $\mathbf{p H}-\mathbf{1}$ & $\mathbf{p H}-\mathbf{2}$ & $\mathbf{p H}-\mathbf{3}$ & $\mathbf{p H}-4$ & $\mathbf{p H}-\mathbf{5}$ & $\mathbf{p H}-\mathbf{6}$ & $\mathbf{p H}-\mathbf{7}$ & $\mathbf{p H}-\mathbf{8}$ & Média \\
\hline A1 & 7,24 & 7,13 & 7,11 & 7,07 & 7,19 & 7,09 & 7,15 & 7,01 & $\mathbf{7 , 1 2}$ \\
\hline A2 & 4,03 & 4,31 & 4,21 & 4,13 & 4,08 & 4,00 & 4,24 & 4,14 & $\mathbf{4 , 1 4}$ \\
\hline A3 & 7,90 & 8,20 & 8,20 & 8,43 & 8,10 & 8,42 & 8,51 & 8,22 & $\mathbf{8 , 2 5}$ \\
\hline
\end{tabular}

Pode-se perceber que a amostra A2 tem um caráter ácido, diferentemente das amostras A1 e A3, com $\mathrm{pH}$ 's praticamente neutros.

$\mathrm{Na}$ análise microbiológica, todos as amostras apresentaram coloração amarelada, sem modificação após submissão da lâmpada UV (Figura 1), demonstrando resultado positivo para presença de coliformes totais e negativo para presença de coliformes fecais, o que é considerado um resultado aceitável para água de consumo humano.

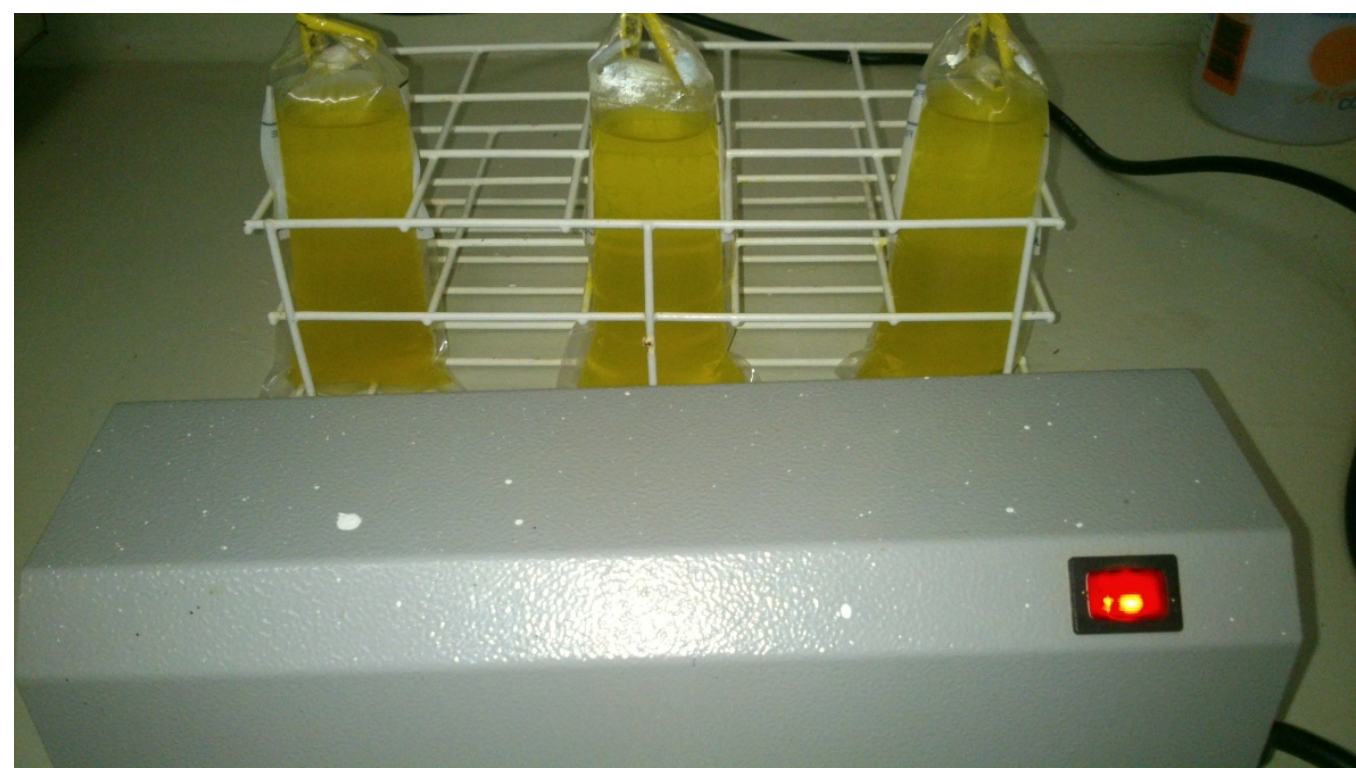

Figura 1 - Inspeção visual sob a luz UV para detecção de coliformes fecais. 
Quanto a alcalinidade da água, encontrou-se as concentrações de íons hidrônio e íons carbonato iguais a zero em todas as amostras, pois com a adição de fenolftaleína (indicador de acidez e basicidade) as amostras continuaram incolores, indicando acidez no meio, portanto ausência de íons hidrônio $\left(\mathrm{OH}^{-}\right)$ e íons carbonato $\left(\mathrm{CO}_{3}{ }^{2-}\right)$, de acordo com a tabela de classificação da alcalinidade da água (Tabela 2).

Tabela 2 - Tabela para avaliar a alcalinidade da água.

\begin{tabular}{cccc}
\hline Resultados & \multicolumn{3}{c}{ Alcalinidades } \\
\hline $\mathbf{P}=\mathbf{0}$ & $\mathbf{O H}^{-}$ & $\mathbf{C O}_{3}{ }^{2-}$ & $\mathbf{H C O}_{3^{-}}$ \\
\hline $\mathbf{P}<\mathbf{M} / \mathbf{2}$ & Zero & Zero & $\mathrm{M}$ \\
$\mathbf{P}=\mathbf{M} / \mathbf{2}$ & Zero & $2 \mathrm{P}$ & $\mathrm{M}-2 \mathrm{P}$ \\
$\mathbf{P}>\mathbf{M} / \mathbf{2}$ & Zero & $2 \mathrm{P}=\mathrm{M}$ & Zero \\
\hline $\mathbf{P}=\mathbf{M}$ & $2 \mathrm{P}-\mathrm{M}$ & $2(\mathrm{M}-\mathrm{P})$ & Zero \\
\hline & $\mathrm{M}$ & Zero & Zero \\
\hline
\end{tabular}

Fazendo uso da equação (1), pode-se obter o valor de $\mathrm{M}$, que será a concentração de $\mathrm{HCO}_{3}{ }^{-}$:

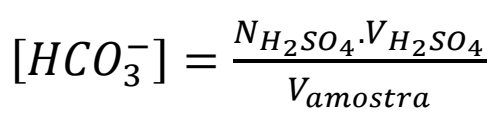

Onde $\mathrm{N}_{\mathrm{H}_{2} \mathrm{SO}_{4}}$ corresponde à normalidade do ácido sulfúrico, $\mathrm{V}_{\mathrm{H}_{2} \mathrm{SO}_{4}}$ é o volume gasto na titulação e $V_{\text {amostra }}$ o volume da amostra.

Logo, na Tabela 3 são apresentados os valores de ácido sulfúrico gastos na titulação e os valores correspondentes da concentração de $\mathrm{HCO}_{3}{ }^{-}$. Pode-se perceber através da mesma que a água do município de Picuí encontrava-se com uma alcalinidade superior às demais.

Tabela 3 - Valores referentes à análise de alcalinidade.

\begin{tabular}{|c|c|c|c|}
\hline & & $\begin{array}{c}V_{\mathrm{H}_{2} \mathrm{SO}_{4}} \\
(\mathrm{~L})\end{array}$ & $\begin{array}{c}{\left[\mathrm{HCO}_{3}^{-}\right]} \\
(\mathrm{Eq} / \mathrm{L})\end{array}$ \\
\hline \multirow[t]{4}{*}{ A1 } & Repetição - 1 & 0,0017 & 0,0014 \\
\hline & Repetição - 2 & 0,0017 & 0,0014 \\
\hline & Repetição - 3 & 0,0018 & 0,0014 \\
\hline & Média & & 0,0014 \\
\hline \multirow[t]{4}{*}{ A2 } & Repetição - 1 & 0,0006 & 0,00048 \\
\hline & Repetição - 2 & 0,0006 & 0,00048 \\
\hline & Repetição - 3 & 0,0006 & 0,00048 \\
\hline & Média & & 0,00048 \\
\hline \multirow[t]{4}{*}{ A3 } & Repetição - 1 & 0,0109 & 0,0087 \\
\hline & Repetição - 2 & 0,0104 & 0,0083 \\
\hline & Repetição - 3 & 0,0105 & 0,0084 \\
\hline & Média & & 0,0085 \\
\hline
\end{tabular}

Para o cálculo da dureza da água, utilizou-se a equação (2) e (3), a fim de se calcular a concentração de carbonato de cálcio.

$$
\begin{gathered}
N_{\text {amostra }} \cdot V_{\text {amostra }}=N_{E D T A} \cdot V_{E D T A} \\
{\left[\mathrm{CaCO}_{3}\right]=N_{\text {amostra }} \cdot \text { eq. } \cdot g_{\mathrm{CaCO}_{3}} \cdot 10^{3}}
\end{gathered}
$$


Onde $N_{\text {amostra }}$ corresponde à normalidade da amostra, $N_{E D T A}$ a normalidade do EDTA, $V_{E D T A}$ o volume de EDTA gasto na titulação e $\left[\mathrm{CaCO}_{3}\right]$ a concentração de carbonato de cálcio. A Tabela 4 contém os valores de EDTA gastos na titulação e os valores correspondentes da concentração de carbonato de cálcio.

Tabela 4 - Valores referentes à análise de dureza da água.

\begin{tabular}{|c|c|c|c|}
\hline & & $\begin{array}{l}V_{E D T A} \\
(\mathrm{~L})\end{array}$ & $\begin{array}{c}{\left[\mathrm{CaCO}_{3}\right]} \\
(\mathrm{mg} / \mathrm{L})\end{array}$ \\
\hline \multirow[t]{4}{*}{ A1 } & Repetição - 1 & 0,002 & 100 \\
\hline & Repetição - 2 & 0,002 & 100 \\
\hline & Repetição - 3 & 0,0019 & 95 \\
\hline & Média & & 98,3 \\
\hline \multirow[t]{4}{*}{ A2 } & Repetição - 1 & 0,001 & 50 \\
\hline & Repetição - 2 & 0,001 & 50 \\
\hline & Repetição - 3 & 0,0008 & 40 \\
\hline & Média & & 46,7 \\
\hline \multirow[t]{4}{*}{ A3 } & Repetição - 1 & 0,0037 & 185 \\
\hline & Repetição - 2 & 0,004 & 200 \\
\hline & Repetição - 3 & 0,0034 & 170 \\
\hline & Média & & 185 \\
\hline
\end{tabular}

Com o auxílio da Tabela 5, pode-se afirmar que a amostra referente ao município de Campina Grande trata-se de uma água 'moderadamente dura', a do município de Cuité corresponde a uma água 'macia' e a de Picuí uma água 'dura'. Todas encontraram-se dentro do limite aceito de até 500 mg/L de $\mathrm{CaCO}_{3}$.

Tabela 5 - Classificação da dureza da água.

\begin{tabular}{cc}
\hline $\begin{array}{c}\text { Carbonato de Cálcio } \\
(\mathbf{m g} / \mathbf{L})\end{array}$ & Classificação de Dureza da Água \\
$<60$ & Macia \\
\hline $60-150$ & Moderadamente dura \\
\hline $150-300$ & Dura \\
\hline$>300$ & Muito dura \\
\hline
\end{tabular}

\section{Conclusão}

Através da análise biológica foi possível afirmar que todas as amostras sugerem águas com potencialidade para uso humano, visto que nenhuma apresentou coliforme fecais. Os $\mathrm{pH}$ das mesmas apresentaram-se neutros, com exceção da água proveniente do município de Cuité, caracterizada como ácida. Quanto à dureza, houve uma variabilidade de resultados nas amostras analisadas, mas permanecendo dentro do padrão estabelecido, com valores menores que $500 \mathrm{mg} / \mathrm{L} \mathrm{CaCO}_{3}$. A alcalinidade foi bastante distinta de uma amostra para outra, complementando a análise de dureza de água.

\section{Agradecimento}

Os autores desse trabalho agradecem à Universidade Federal de Campina Grande e a Unidade Acadêmica de Engenharia Química. 


\section{Chemical and microbiological water analysis in different collection points}

Albstract: The water is one of the main requeriments for the existence and maintenance of planet's life. Despite its abundance, the most of available water is not suitable for drinking, since natural reasons like excess salt until reasons caused by human like the pollution. This pollution is sometimes perceptible, however the largely is only discovered through specific and accurate analysis. Many public agencies aim to control the water potability supplied in home networks, establishing parameters to be met. This present work aimed ckeck if the water supplied in the cities of Campina Grande, Cuité and Picuí, located in the Paraiba state, corresponded qualitatively to the expected. Therefore, it was analyzed physical and chemical factors like $\mathrm{pH}$, alkalinity and water hardness, hrough specific devices and titulometry techniques. Microbiological factors were also analyzed to verify the presence of total coliforms and fecal coliforms. All samples followed by a sterile collection process and showed tolerable levels for the punctuated factors, being suitable for domestic use.

Keywords: water, potability, alkalinity, microbiological analysis

\section{Referências bibliográficas}

Comissão Especializada da Qualidade da Água. Acesso em: 27 de Setembro de 2015. Disponível em: http://www.apda.pt/site/upload/FT-QI-10-\%20Dureza\%20total.pdf.

CONFALONIERI, Ulisses; HELLER, Léo; AZEVEDO, Sandra. Água e Saúde: Aspectos Globais e Nacionais. Acesso em: 27 de Setembro de 2015. Disponível em: http://www.abc.org.br/IMG/pdf/doc808.pdf

DIAS, Maria Fernanda Falcone. Qualidade Microbiológica de Águas Minerais em Garrafas Individuais Comercializadas em Araraquara-SP, Dissertação de Mestrado, Universidade Estadual Paulista, 66 pág.: 2008.

LEE, John David. Química inorgânica não tão concisa, Tradução da $5^{a}$ ed. Inglesa. Editora Edgard Blücher Ltda. São Paulo: 1999.

NOVAK, Franz Reis; ALMEIDA, João Aprígio Guerra de; ASENSI, Marise Dutra; MORAES, Bianca Aguiar de; RODRIGUES, Dália dos Prazeres. Resistência antimicrobiana de coliformes isolados de leite humano ordenhado. Cad. Saúde Pública, Rio de Janeiro, 2001.

OLIVEIRA, Aline Maxilline Pereira. Acesso em: 27 de Setembro de 2015. Disponível em: http://www.kurita.com.br/adm/download/Alcalinidade_e_Dureza.pdf.

PRÜSS-USTIN, Annette; BOS, Robert; GORA, Fiona; BARTRAM, Jamie. Safer water, better health. Costs, benefits and sustainability of interventions to protect and promote health. World Health Organization. Geneva: 2008. 